\title{
Phraseology in pathology reports. A comparative study of interpretation among pathologists and surgeons
}

\author{
R L Attanoos, A D Bull, A G Douglas-Jones, L J Fligelstone, D Semararo
}

\begin{abstract}
This questionnaire based study compared the interpretation, use and preferences, among pathologists and surgeons, of descriptive phrases found in surgical reports. The results show that there is a wide variation in individual interpretation of phrases in both groups. The frequency of usage of phrases by pathologists and preference for phrases by surgeons were also diverse. The adoption of a limited number of descriptive phrases that are mutually understood and acceptable for use by both pathologists and clinicians is recommended to avoid interpretive ambiguity in pathology reports.

(f Clin Pathol 1996;49:79-81)
\end{abstract}

Keywords: surgical reports, phraseology, communication.

Surgical reports should provide clinicians with diagnostically accurate and medically useful information with which to effect the best management of patients. Moreover, the information should be presented in an unambiguous manner. In practice, pathologists use a variety of descriptive phrases to convey to the clinician their level of certainty in making a particular diagnosis. The interpretation of these phrases and the level of certainty they convey is likely to be subjective and may have implications for patient management. Current audit schemes in histopathology ${ }^{12}$ aim to provide useful reports, but do not address this problem. The aim of this study was to make a comparative assessment of interpretation and use, by both pathologists and surgeons, of the common descriptive phrases found in surgical reports.

\footnotetext{
Methods

A questionnaire was constructed using the most commonly used descriptive phrases found among 300 randomly selected surgical reports from the 1994 files of the Department of Histopathology, University Hospital of Wales. The phrases were: 'diagnostic of', 'that of', 'show', 'characteristic of', 'indicative of', 'represent', 'in keeping with', 'consistent with', 'highly sug-
}

gestive of', 'favour', 'suggestive of', 'suspicious of', and 'reminiscent of'. The Concise Oxford Dictionary was used to establish the exact definition of each phrase. The questionnaire was distributed to 20 pathologists and 20 surgeons within the University Hospital and to three local district general hospitals. All staff were of registrar, senior registrar or consultant grade. In each group the participants were asked to score each phrase on a scale from 0 (conveying total diagnostic uncertainty) to 5 (conveying total diagnostic certainty). In addition, for each phrase, pathologists were asked to comment on their frequency of usage in three categories (common, uncommon, never) and surgeons on their preferences in two categories (like, dislike/ confusing).

\section{Results}

Six of the 13 descriptive phrases ('diagnostic of', 'that of', 'show', 'characteristic of', 'indicative of', and 'represent') are semantically definitive terms which should only be used to communicate total certainty in diagnosis (a score of 5). The other seven phrases are subject to variability in interpretation. The level of diagnostic certainty conveyed by the phrases as interpreted by pathologists and surgeons is illustrated in table 1. For the definitive phrases, the considerable variation in interpretation between both groups and individuals is highlighted. For example, the term 'diagnostic of' conveyed more diagnostic certainty to pathologists than to surgeons. Table 1 also shows that for the less well defined terms there is even more disagreement. Table 2 shows the variation in the pathologists' use of phrases and the surgeons' preference for phrases. With the exception of 'diagnostic of' and 'that of', used by $50 \%$ and $95 \%$ of pathologists, respectively, the other definitive terms preferred by surgeons were infrequently used ('show' (35\%), 'characteristic of' $(25 \%)$, 'indicative of' $(20 \%)$, and 'represent' (20\%)). In comparison, the terms most commonly used by pathologists - in keeping with' (85\%), 'consistent with' (90\%), 'those of' (95\%), and 'suggestive of' $(80 \%)-$ were regarded as disliked/confusing terms by, respectively, $65,30,45$, and $70 \%$ of surgeons. 
Table 1 Scoring of phases

\begin{tabular}{|c|c|c|c|c|c|c|}
\hline \multirow[b]{2}{*}{ Phrase } & \multicolumn{6}{|c|}{ Score } \\
\hline & 0 & 1 & 2 & 3 & 4 & 5 \\
\hline \multicolumn{7}{|l|}{ Definitive terms } \\
\hline \multicolumn{7}{|l|}{ Diagnostic of } \\
\hline surgeon & & & 1 & & 4 & 15 \\
\hline \multicolumn{7}{|l|}{ That of } \\
\hline pathologist & & & 1 & 1 & 3 & 15 \\
\hline surgeon & 1 & & 4 & 2 & 5 & 8 \\
\hline \multicolumn{7}{|l|}{ Show } \\
\hline pathologist & & & 1 & 1 & 3 & 15 \\
\hline surgeon & & 1 & 2 & 3 & 4 & 10 \\
\hline \multicolumn{7}{|l|}{ Characteristic of } \\
\hline pathologist & & & & 2 & 7 & 11 \\
\hline \multirow{2}{*}{\multicolumn{7}{|c|}{ Indicative of }} \\
\hline & & & & & & \\
\hline pathologist & & & 1 & 3 & 5 & 11 \\
\hline surgeon & & 1 & 1 & 2 & 12 & 4 \\
\hline \multicolumn{7}{|l|}{ Represent } \\
\hline pathologist & & & 1 & 3 & 6 & 11 \\
\hline surgeon & & 2 & 2 & 2 & 6 & 9 \\
\hline \multicolumn{7}{|l|}{ Non-definitive terms } \\
\hline \multicolumn{7}{|l|}{ In keeping with } \\
\hline pathologist & & & 2 & 7 & 11 & \\
\hline surgeon & & & 5 & 7 & 6 & 2 \\
\hline \multicolumn{7}{|l|}{ Consistent with } \\
\hline pathologist & & & 1 & 8 & 11 & \\
\hline surgeon & & & 1 & 10 & 7 & 2 \\
\hline \multicolumn{7}{|l|}{ Highly suggestive of } \\
\hline pathologist & & & & 4 & 16 & \\
\hline surgeon & & & 1 & 4 & 15 & \\
\hline \multicolumn{7}{|l|}{ Favour } \\
\hline pathologist & & & 2 & 13 & 5 & \\
\hline surgeon & & 1 & 6 & 8 & 5 & \\
\hline \multicolumn{7}{|l|}{ Suggestive of } \\
\hline pathologist & & & 5 & 15 & & \\
\hline surgeon & & 1 & 2 & 16 & 1 & \\
\hline \multicolumn{7}{|l|}{ Suspicious of } \\
\hline pathologist & & 1 & 6 & 10 & 3 & \\
\hline surgeon & & 1 & 6 & 9 & 4 & \\
\hline \multicolumn{7}{|l|}{ Reminiscent of } \\
\hline pathologist & 2 & 4 & 8 & 5 & 1 & \\
\hline surgeon & 4 & 5 & 8 & 2 & 1 & \\
\hline
\end{tabular}

\section{Discussion}

This study is, to our knowledge, the first to assess the problems of histopathologists attempting to convey their uncertainty in pathological diagnosis to their clinical colleagues through the written word in reports. The results show that a variety of qualifying terms are used, the meanings of which are interpreted differently by pathologists and surgeons, conferring potential ambiguity to histopathology reports. The variable interpretation of the six definitive phrases used by pathologists infers an inherent misunderstanding and consequent misapplication of these terms in surgical reports. It is also apparent that surgeons prefer definitive terms relating to unequivocal diagnoses with which to plan patient management, whereas pathologists prefer to issue reports using less definitive phrases. The term 'diagnostic of' was found to be the least ambiguous in interpretation in both groups; however, only $30 \%$ of pathologists used the term commonly, despite its favour among surgeons. The increasing problem of medicolegal challenge to any diagnostic opinion may well induce considerable caution in the use of certain phrases in reports and this may indeed explain the pathologists' reluctance to use such a definitive term as 'diagnostic of', which semantically conveys total certainty with respect to diagnosis and permits no room for manoeuvre in any litigious procedure. Pathologists may have to make a qualified diagnosis for a number of reasons such as size and preservation of biopsy tissue, non-specificity or atypicality of histological appearances and incomplete clinical information. As for any investigation, the results must be interpreted in the context of the whole clinical picture. Most routine pathological interpretation takes place in the context of incomplete clinical information and this leads to diagnostic uncertainty. If the histology is reported in the light of other findings (clinical examination, blood tests or imaging) or after discussion with the referring clinician, the diagnostic confidence of the pathologist is likely to be higher. In most district general hospitals pathologists and clinicians know each other well and communicate frequently and in many cases it is likely that ad hoc agreements on mutually recognised phrases used by the pathologist in his reports indicate a particular degree of certainty to the clinician. If the clinician is certain he understands the meaning of the report, then it will give him the confidence to base management decisions on the written word. This degree of understanding may be more difficult to establish in large teaching hospitals where there are numerous rotating junior staff and where senior staff have many other responsibilities. In cytological practice the ambiguities of language have being minimised by the adoption of a numerical reporting system which encompasses levels of diagnostic uncertainty. In the National Breast Screening Programme, ${ }^{4}$ the Royal College of Pathologists Working Committee recommended the following classification: $\mathrm{C} 1$ /inadequate; $\mathrm{C} 2 / \mathrm{be}-$ nign; C3/suspicious, probably benign; C4/ suspicious, probably malignant; and $\mathrm{C} 5 / \mathrm{malig}$ nant. In this context the term 'suspicious' is used to convey that the sample examined contains material 'not absolutely diagnostic of malignancy'. While the use and understanding

Table 2 Pathologists' use of phrases and surgeons' preference for phrases

\begin{tabular}{|c|c|c|c|c|c|}
\hline & \multicolumn{3}{|c|}{ Pathologists' use } & \multicolumn{2}{|c|}{ Surgeons' preferences } \\
\hline & Common & Uncommon & Never & Like & Dislike/confusing \\
\hline Diagnostic of & $10(50 \%)$ & $8(40 \%)$ & $2(10 \%)$ & $20(100 \%)$ & 0 \\
\hline That of & $19(95 \%)$ & $1(5 \%)$ & & $11(55 \%)$ & $9(45 \%)$ \\
\hline Show & $7(35 \%)$ & $8(40 \%)$ & $5(25 \%)$ & $15(75 \%)$ & $5(25 \%)$ \\
\hline Characteristic of & $5(25 \%)$ & $10(50 \%)$ & $5(25 \%)$ & $15(75 \%)$ & $5(25 \%)$ \\
\hline Indicative of & $4(20 \%)$ & $7(35 \%)$ & $9(45 \%)$ & $14(70 \%)$ & $6(30 \%)$ \\
\hline Represent & $4(20 \%)$ & $10(50 \%)$ & $6(30 \%)$ & $13(65 \%)$ & $7(35 \%)$ \\
\hline In keeping with & $17(85 \%)$ & $3(15 \%)$ & 0 & $7(35 \%)$ & $13(65 \%)$ \\
\hline Consistent with & $18(90 \%)$ & $2(10 \%)$ & 0 & $14(70 \%)$ & $6(30 \%)$ \\
\hline Highly suggestive of & $10(50 \%)$ & $9(45 \%)$ & $1(5 \%)$ & $11(55 \%)$ & $9(45 \%)$ \\
\hline Favour & $5(25 \%)$ & $12(60 \%)$ & $2(10 \%)$ & $4(20 \%)$ & $16(80 \%)$ \\
\hline Suggestive of & $15(75 \%)$ & $5(25 \%)$ & 0 & $5(25 \%)$ & $15(75 \%)$ \\
\hline Suspicious of & $6(30 \%)$ & $13(65 \%)$ & $1(5 \%)$ & $6(30 \%)$ & $14(70 \%)$ \\
\hline Reminiscent of & 0 & $8(40 \%)$ & $12(60 \%)$ & $1(5 \%)$ & $19(95 \%)$ \\
\hline
\end{tabular}


of the term 'suspicious' is defined in cytological practice, the use and interpretation of all the non-definitive phrases among both pathologists and surgeons showed considerable disparity, highlighting the necessity for some limitation, in the use of these and the other definitive terms. Phrases should be mutually understood and accepable for use by both pathologists and clinicians. To this end, the use of only one of the six definitive phrases in current use is suggested as all semantically convey the same level of certainty. A recommendation for use of a limited repertoire of non-definitive phrases is more difficult to achieve as no single nondefinitive term clearly conveys a greater or lesser degree of certainty than any other nondefinitive term. National guidelines for the use of phraseological terms in histopathological reporting may be required to address such a problem as there is a clear necessity for nondefinitive usage in reports.
In practice, histopathologists are part of a clinical team and regular clinicopathological meetings allow an exchange of information to take place on selected cases. Such meetings also enable the clinician to understand the source of diagnostic difficulties for the pathologist, preventing interpretive ambiguity and facilitating optimal patient management. Offsite private pathology services do not facilitate close contact between pathologists and clinicians and if market forces effect their expansion, the need for unambiguous surgical reports is greater now than ever.

1 Ramsay AD, Gallagher PJ. Local audit of surgical pathology. Am f Surg Pathol 1992;16:476-82.

2 Campbell F, Griffiths DFR. Quantitative audit of the content of histopathology reports. $\mathcal{f}$ Clin Pathol 1994;47:360-1.

3 Allen RE (ed). The Concise Oxford Dictionary of Current English. 8th edn. Oxford: Clarendon Press Publications, 1990.

4 Department of Health and Royal College of Pathologists Working Group. Pathology Reporting in Breast Cancer Screening Draft Guidance. London: Royal College of Pathologists, 1989.

\title{
Tumour related cutaneous elastophagocytosis
}

\author{
E C Sweeney, M McDermott
}

\begin{abstract}
Dermal granulomatous inflammation was identified immediately adjacent to seven (77\%) of nine atypical fibroxanthomas arising in sun damaged skin. Concomitant elastophagocytosis was observed in five $(56 \%)$ of these seven patients. Similar inflammation with elastophagocytosis was found in association with only two $(6 \%)$ of 36 epithelial tumours arising on the same background (10 basal and 10 squamous cell carcinomas, 10 nodular malignant melanomas, and six keratoacanthomas). Granulomatous inflammation is an unusual dermal reaction to tumour and elastophagocytosis is rare. The fact that both of these features occur with inordinate frequency in association with atypical fibroxanthomas, when compared with other, more common skin tumours, suggests that atypical fibroxanthomas might modulate the inflammatory response, either passively, by its dermal location, or actively, by secreting locally effective cytokines.

(f Clin Pathol 1996;49:81-83)
\end{abstract}

Keywords: solar elastosis, granulomatous inflammation, elastophagocytosis, skin carcinoma, atypical fibroxanthoma.

Phagocytosis of dermal elastotic material is a well recognised feature of many inflammatory skin disorders and is a defining feature of actinic granuloma. Some investigators believe that the process of elastophagocytosis is a reaction to collagen that has been damaged by ultraviolet light. ${ }^{1}$ Others, however, maintain that it is nonspecifically associated with local inflammation. ${ }^{2}$

Many skin tumours evoke inflammation and a concurrent granulomatous reaction is thought not to be unusual by some dermatopathologists. Support for this claim, however, appears to be largely anecdotal. Our attention was drawn to this peculiar reaction by the observation of extensive elastophagocytosis adjacent to an atypical fibroxanthoma.

This study was undertaken to ascertain the frequency with which elastophagocytosis occurs in association with tumours arising in sun damaged skin and whether or not the type of tumour involved has any bearing on its occurrence.

\section{Methods}

Eleven cases of atypical fibroxanthoma were retrieved from archival material stored over a 25 year period. Two of these had arisen in skin that had not been exposed to the sun and therefore were excluded from the study. Similarly, five cases of dermatofibrosarcoma protuberans and 15 dermatofibromas had no evidence of surrounding solar elastosis and were also excluded. However, 10 cases of invasive basal cell carcinoma (BCC), 10 of squamous cell carcinoma (SCC), 10 of nodular malignant melanoma (NMM), and six of keratoacanthoma were included. The nine atypical 